\title{
Pobreza, jóvenes y consumo de tabaco en México
}

\author{
Carl Reddy-Jacobs, MB, ChB, MSc, (1) Martha Ma.Téllez-Rojo, M en C, Dr en C,(2) \\ Fernando Meneses-González, M en C, MSP, ${ }^{(2)}$ Julio Campuzano-Rincón, MD, MSP, Dr en C, ${ }^{(2)}$ \\ Mauricio Hernández-Ávila, MC, PhD. ${ }^{(2)}$
}

\section{Reddy-Jacobs C, Téllez-Rojo MM, Meneses-González F, Campuzano-Rincón J, Hernández-Ávila M. Pobreza, jóvenes y consumo de tabaco en México. Salud Publica Mex 2006;48 supl I:S83-S90.}

\section{Resumen}

Objetivo. Caracterizar el consumo de tabaco de acuerdo con el nivel de pobreza en una muestra probabilística nacional de jóvenes de 10 a 21 años de edad que viven en zonas suburbanas de México. El estudio forma parte de la evaluación del programa gubernamental Oportunidades (200I). Material y métodos. Se entrevistaron a 29548 jóvenes residentes en 30000 hogares. Se calcularon la prevalencia de fumar y los factores relacionados que diferencian al fumador habitual del experimentador. Resultados. La prevalencia de fumadores y experimentadores fue de $3.5 \%$ (IC95\% 3.3-3.7) y $9.9 \%$ (IC95\% 9.6-I0.2), respectivamente. El nexo entre los diferentes factores que se vinculan con las condiciones de fumador y experimentador se evaluó a través de modelos de regresión logística para datos agrupados. Después de ajustar por nivel de pobreza del hogar y consumo de alcohol y drogas, se detectó una relación significativa $(R M=I .5, p<0.01)$ con tener un trabajo remunerado y una diferencial entre el género y el grupo de edad. Conclusiones. La disponibilidad de dinero por parte del joven es un factor que contribuye a explicar la transición entre experimentar y fumar. Dado que el programa Oportunidades incluye transferencias monetarias a los hogares incorporados al programa, es necesario instituir campañas de prevención para evitar que la disponibilidad adicional de dinero en los jóvenes se utilice en la compra de cigarillos.

Palabras clave: consumo de tabaco; adolescentes; pobreza; Oportunidades; México
Reddy-Jacobs C, Téllez-Rojo MM, Meneses-González F, Campuzano-Rincón J, Hernández-Ávila M.

Poverty, youth and consumption

of tobacco in Mexico.

Salud Publica Mex 2006;48 suppl I:S83-S90.

\section{Abstract}

Objective:To characterize tobacco use according to level of poverty in a random, nationally representative sample of adolescents ( 10 to 21 years old), living in urban areas with less than 50000 inhabitants. The study was done in $200 \mathrm{I}$ as part of the baseline assessment of the evaluation of the governmental program, Oportunidades. Material and methods. A questionnaire was applied to 29548 adolescents living in 30000 selected households and it included specific questions on individual tobacco use among other questions. Results. The prevalence of smokers was 3.5\% (95\% Cl: $3.3 \%-3.7 \%$ ) and experimenters $9.9 \%$ (95\% Cl: $9.6 \%-10.2 \%)$. A logistic regression model for clustered data was constructed in order to evaluate the associated factors that distinguish a smoker from an experimenter.After adjusting for level of poverty of the household and use of alcohol and drugs, a significant association $(O R=1.5, p<0.0 \mathrm{I})$ was found with having a paid job and a differential association was found between gender and age group. Conclusions. The results of this study suggest that the additional availability of money that an adolescent has, could increase the prevalence of tobacco smoking and that the program Oportunidades should include prevention campaigns directed specifically at this population group.

Key words: tobacco consumption; adolescents; poverty; Mexico

(I) Unit for Clinical and Biomedical TB Research, Medical Research Council. Durban, South Africa

(2) Centro de Investigación en Salud Poblacional, Instituto Nacional de Salud Pública. Cuernavaca, Morelos, México

Fecha de recibido: 4 de noviembre de 2005 - Fecha de aprobado: 25 de abril de 2006 Solicitud de sobretiros: Dra. Martha Ma. Téllez-Rojo. Instituto Nacional de Salud Pública. Av. Universidad 655. Col. Sta. Ma. Ahuacatitlán, 62508 Cuernavaca, Morelos, México.

Correo electrónico: mmtellez@insp.mx 
$\mathrm{E}^{\mathrm{I}}$ consumo de tabaco es en la actualidad uno de los principales problemas de salud pública mundial; para el periodo de 2025 a 2030 se calcula que en países en desarrollo morirán cerca de siete millones de personas por daños a la salud atribuibles al consumo de tabaco. ${ }^{1}$ Aun cuando se han realizado múltiples esfuerzos para reducir el consumo de tabaco, la prevalencia de éste varía entre los diferentes países o regiones; en algunos de ellos ${ }^{2,3}$ permanece estable respecto al tiempo y en otros muestra variaciones muy amplias. ${ }^{4,5} \mathrm{El}$ mercado de tabaco en la población adulta tiene su origen en la prevalencia del consumo de tabaco en jóvenes y lo promueve la industria del tabaco dentro de este grupo. ${ }^{6,7}$ Es posible reconocer la presencia de una paradoja en el consumo de tabaco en esta población; por un lado, en países desarrollados en los que la prevalencia de consumo en jóvenes disminuye pese a que el ingreso per cápita es mayor, también en esas mismas naciones se observa una disminución del consumo conforme aumenta el ingreso y el nivel educativo de los jóvenes; por el contrario, en países en desarro1lo, donde dicho ingreso es menor, la prevalencia tiene una tendencia creciente ${ }^{8,9}$ y se vincula en grado significativo con el ingreso y el nivel educativo: entre mayores sean éstos, más alta es la prevalencia del consumo. En México, la tendencia en el consumo de tabaco en jóvenes es ascendente y una expresión directa de ello es la disminución de la edad promedio de inicio que se ha reducido de 21.8 a 13.2 años en cinco décadas. ${ }^{10}$ La prevalencia de consumo de tabaco en el joven mexicano tiene una relación directa y positiva con el nivel socioeconómico familiar: este trabajo explora la presencia de la paradoja entre consumo de tabaco e incremento de la prevalencia de tabaquismo en un sector marginado que se desarrolla en condiciones de pobreza en las zonas suburbanas del país.

\section{Material y métodos}

Se realizó un estudio transversal a partir de la información de 29548 jóvenes recolectada en la encuesta de evaluación del Programa de Desarrollo Humano Oportunidades (en adelante, Oportunidades) realizada en el año 2001. Esta información procede de una encuesta probabilística aplicada en 30000 hogares de localidades suburbanas (definidas como áreas con poblaciones de 2500 a 50000 habitantes) ubicadas en zonas de alta marginación. El protocolo de evaluación lo aprobó el Comité de Ética del Instituto Nacional de Salud Pública. Se definió como población joven a los residentes cuyas edades eran de 10 a 21 años y con carta de consentimiento firmada, se les aplicó un cuestionario. Este incluyó diversas variables: a) sociodemográficas (edad, sexo, estado civil); b) educativas (asistencia y tipo de escuela, desempeño escolar, ayuda de los padres con tareas, ocupación, trabajo remunerado y servicios gratuitos en la comunidad); c) bienestar subjetivo (satisfacción con la vida y respuesta a una crisis); y d) adicciones (tabaquismo, alcoholismo y consumo de drogas ilegales). Para definir el nivel de pobreza del hogar se utilizó el índice de pobreza que recomienda el propio programa Oportunidades. ${ }^{11}$ En la construcción de este indicador se emplearon variables como sexo y escolaridad del jefe del hogar, gasto común, características de vivienda (material del piso, número de cuartos, baño, agua entubada) y enseres en el hogar (refrigerador, lavadora, automóvil, estufa de gas), accesibilidad a la seguridad social y región geográfica de residencia. Con base en los puntos de corte preestablecidos en este indicador de pobreza, los hogares se clasificaron en "hogares no pobres", "hogares cercanos a la línea de pobreza" y "hogares pobres".

De acuerdo con el hábito tabáquico, se definió como experimentador al joven que contestó de forma afirmativa a la pregunta: ¿Has fumado cigarros alguna vez en tu vida, aunque hayan sido una o dos fumadas o bocanadas? y que negara fumar en la actualidad. Se consideró fumador actual aquel que contestó sólo de forma afirmativa a la pregunta: ¿Fumas actualmente?; y, por último, se consideró como no fumador a quien contestó de modo negativo ambas preguntas. La disponibilidad líquida de dinero se definió como proxy mediante la variable de pago por un trabajo remunerado la semana anterior a la entrevista, siempre que esa remuneración representara por lo menos una hora de trabajo.

\section{Análisis estadístico}

Se calcularon prevalencias generales y específicas de consumo de tabaco para algunos subgrupos de interés (edad, género, nivel de pobreza y trabajo remunerado). Se evaluó la vinculación bivariada entre la condición de tabaquismo (no fumadores, experimentadores y fumadores actuales) y variables posiblemente relacionadas, entre las que destacan grupo etáreo, género, nivel de pobreza del hogar, tipo de escuela a la que asiste, trabajo remunerado y asistencia a la escuela. Para estas dos últimas variables se realizó un análisis estratificado por edad. La relevancia estadística de los nexos entre variables categóricas y hábito tabáquico se realizó a través de la prueba ji cuadrada. Para la diferencia de medias de variables continuas que no cumplieron con el supuesto de normalidad para el consumo de tabaco se recurrió a la prueba no paramétrica de Kruskal Wallis. ${ }^{12,13}$ Para evaluar la relación multivariada entre estos factores y la condición de tabaquismo 
(no fumador/experimentador y experimentador/fumador), se generaron modelos de regresión logística para datos agrupados en los que la correlación derivada de la residencia en una misma localidad se incorporó al definir la localidad como un conglomerado. Para explorar los diferentes factores vinculados con los diferentes niveles de pobreza se realizó un análisis estratificado por condición de pobreza del hogar mediante los grupos definidos por Oportunidades (no pobre, cercano a la línea de pobreza, pobre). Los modelos propuestos se evaluaron a través de técnicas diagnósticas estándar para regresión logística (pruebas de bondad de ajuste e influencia, así como análisis de residuos). El análisis de la información se llevó a cabo con el paquete estadístico STATA 7.0 (Stata Corp.).

\section{Resultados}

La distribución por sexo de la población de estudio fue homogénea con una mayor participación de jóvenes entre 10 y 14 años de edad. El 68.3\% de la muestra se clasificó como perteneciente a hogares pobres; $20.6 \%$ a hogares cercanos a la línea de pobreza; y $11.1 \%$ a hogares no pobres con una distribución por sexo muy similar entre los grupos. El 15.2\% de los jóvenes menores de 18 años de edad señaló tener trabajo remunerado $(19.8 \%$ en hombres contra $10.7 \%$ en mujeres, $p<0.01)$ y $12.1 \%$ de los jóvenes de 18 años o mayores notificaron esta actividad (15.7\% en hombres contra 8.6\% en mujeres, $p<0.01$ ) (cuadro I).

La prevalencia de fumadores y experimentadores fue de $3.5 \%$ (IC95\% 3.3-3.7) y 9.9\% (IC95\% 9.6-10.2), respectivamente. Para el grupo de 19 a 21 años la prevalencia de fumadores y experimentadores fue mayor: $9.4 \%$ y $18.1 \%$, respectivamente $(p<0.05)$ en relación con los grupos más jóvenes. En la comparación por género, las mujeres informaron una prevalencia de fumadoras y experimentadoras menor a la registrada por los hombres (1.2\% contra $6.2 \%$ y $8 \%$ contra $12.2 \%$ para fumadores y experimentadores, respectivamente). La prevalencia de fumadores actuales y experimentadores disminuyó conforme aumentó el valor del índice de pobreza del hogar: $4.9,4.7$ y $2.9 \%$ en las categorías de hogares no pobres, hogares cercanos a la línea de pobreza y hogares pobres, respectivamente. Este mismo patrón se presentó en el grupo de experimentadores, si bien con mayores prevalencias $(14.7,11.6$ y $8.6 \%$, respectivamente). Los jóvenes con empleo remunerado y menores de 18 años señalaron una prevalencia cruda de consumo de tabaco siete veces mayor que la prevalencia entre los que no trabajan; por otro lado, esa misma prevalencia en los jóvenes con trabajo remunerado y mayores de 18 años fue cuatro veces mayor respecto de los no trabajadores. La prevalencia cruda de experimentadores también fue mayor entre los jóvenes que trabajan en relación con los que no lo hacen. Tanto el tabaquismo como la experimentación en escolares fueron menores que en el grupo de jóvenes que no asisten a la escuela (cuadro II).

De acuerdo con el modelo de regresión logística multivariado, los principales factores vinculados con ser fumador en comparación con ser experimentador, mediante ajuste con el indicador de pobreza del hogar, fueron el género, el trabajo remunerado y el consumo de alcohol o drogas, o ambos. Se ha notificado una relación diferencial entre el consumo de tabaco y el género según sea la edad de los jóvenes. En el grupo de 19 a 21 años de edad se observó que los hombres tenían 4.3 veces más posibilidades de ser fumadores que las mujeres. La razón de momios (RM) correspondiente para el grupo de edad de 15 a 18 años fue 2.55 y 1.7 en el grupo de edad de 10 a 14 años. Los jóvenes con trabajo remunerado tuvieron 1.5 veces más posibilidad de ser fumadores que experimentadores en comparación con los que no tenían trabajo remunerado. No se observaron diferencias estadísticamente significativas de acuerdo con el grupo de pobreza (cuadro III).

El análisis estratificado de acuerdo con la clasificación de pobreza del hogar (cuadro IV) evidenció que el género y el consumo de alcohol y drogas son factores comunes en los tres estratos: tener un trabajo remunerado se relacionó en los tres grupos con una mayor posibilidad de ser fumador, aunque este nexo fue estadísticamente significativo en el grupo correspondiente a los hogares cercanos a la línea de pobreza y marginalmente significativa en el grupo de hogares clasificados como pobres.

\section{Discusión}

Las prevalencias informadas en esta muestra de jóvenes son menores en comparación con otros estudios en poblaciones de jóvenes que residen sobre todo en localidades urbanas donde las prevalencias varían en alrededor de 20\%. ${ }^{14-15}$ Es posible que las bajas prevalencias detectadas en este trabajo se deban a diferencias metodológicas en la definición de fumador y las características de la población bajo estudio, ya que provienen de zonas urbanas pequeñas (entre 2500 y 50 000 habitantes) con un alto grado de marginación, a diferencia de los estudios antes mencionados llevados a cabo en población urbana o muestras seleccionadas a partir de la población escolarizada.

La población estudiada mostró un nexo entre el consumo de tabaco y el de alcohol y drogas, sobre todo en los jóvenes experimentadores de forma independiente del nivel socioeconómico del hogar. $\mathrm{Al}$ ajustar por este últi- 
Cuadro I

Características socioeconómicas y demográficas de los jóvenes participantes en la evaluación basal del programa Oportunidades (México, 200I)

\begin{tabular}{|c|c|c|c|c|c|c|}
\hline & & & & & & \\
\hline & $n$ & $\%$ & $n$ & $\%$ & $n$ & $\%$ \\
\hline & 14949 & 50.59 & 14599 & 49.41 & 29548 & 100 \\
\hline Edad en años & & & & & & \\
\hline $10-14$ & 7509 & 50.23 & 7676 & 52.58 & 15185 & 51.39 \\
\hline $15-18$ & 4774 & 31.94 & 4659 & 31.91 & 9433 & 31.92 \\
\hline$|9-2|$ & 2666 & 17.83 & 2264 & $|5.5|$ & 4930 & 16.68 \\
\hline Clasificación de pobreza del hogar & & & & & & \\
\hline No pobre & 1683 & 11.26 & | 585 & 10.86 & 3268 & 11.06 \\
\hline Cerca de la línea de pobreza & 3132 & 20.95 & 2959 & 20.27 & 6091 & 20.61 \\
\hline Pobre & 10134 & 67.79 & 10055 & 68.87 & 20189 & 68.33 \\
\hline Con trabajo remunerado & & & & & & \\
\hline$<18$ años & 1606 & 10.74 & 2899 & 19.86 & 4505 & 15.25 \\
\hline$\geq 18$ años & 1288 & 8.62 & 2305 & 15.79 & 3593 & 12.16 \\
\hline Sin trabajo remunerado & II $84 \mid$ & 79.21 & 9097 & 62.31 & 20938 & 70.86 \\
\hline Sin información & 214 & 1.43 & 298 & 2.04 & 512 & 1.73 \\
\hline Asistencia a la escuela & & & & & & \\
\hline$<18$ años & 8291 & 55.46 & 8382 & 57.41 & 16673 & 56.43 \\
\hline$\geq 18$ años & 607 & 4.06 & 572 & 3.92 & 1179 & 3.99 \\
\hline No asiste & 5477 & 36.64 & 5023 & 34.41 & 10500 & 35.54 \\
\hline Sin información & 574 & 3.84 & 622 & 4.26 & 1196 & 4.05 \\
\hline Nivel máximo de estudios & & & & & & \\
\hline Preescolar & 540 & 3.61 & 548 & 3.75 & 1088 & 3.68 \\
\hline Primaria & 7863 & 52.60 & 7964 & 54.55 & 15827 & 53.56 \\
\hline Secundaria & 4685 & 31.34 & 4410 & 30.21 & 9095 & 30.78 \\
\hline Preparatoria & 1517 & 10.15 & I 284 & 8.80 & 2801 & 9.48 \\
\hline Licenciatura & 150 & 1.00 & 118 & 0.81 & 268 & 0.91 \\
\hline Faltantes & 194 & 1.30 & 275 & 1.88 & 469 & 1.59 \\
\hline Estado civil & & & & & & \\
\hline Casado & 1621 & 10.84 & 810 & 5.55 & 2431 & 8.23 \\
\hline Separado & 223 & 1.49 & 45 & 0.31 & 268 & 0.91 \\
\hline Soltero & 9656 & 64.59 & 10320 & 70.69 & 19976 & 67.61 \\
\hline Faltantes & 3449 & 23.07 & 3424 & 23.45 & 6873 & 23.26 \\
\hline
\end{tabular}

mo se documentó un comportamiento diferencial por género y grupo etáreo. Los hombres de 19 a 21 años mostraron 4.25 veces más posibilidad de ser fumadores que las mujeres, mientras que disminuyó a 2.55 y 1.7 en el grupo de 15 a 18 años y 10 a 14 años, respectivamente.

Los resultados de este estudio sugieren que en esta población el factor económico más importante para explicar la transición entre experimentador y fumador es la disponibilidad de dinero por parte del joven. ${ }^{17-19} \mathrm{El}$ análisis multivariado mostró que el nivel de pobreza del hogar no es un factor vinculado con el consumo de tabaco por parte de los jóvenes; pese a ello, resultó positiva la relación entre el consumo de tabaco y la disponibilidad de dinero proveniente de un trabajo remunerado. Esto sugiere que la disponibilidad directa de ingreso es tal vez un factor de mayor peso, en com- paración con el ingreso del hogar, en el sentido de la accesibilidad que pueden tener los jóvenes a la compra de cigarrillos. Estos hallazgos también son consistentes con otros estudios que han encontrado un nexo entre el ingreso personal del joven y su consumo de tabaco.

En el grupo de hogares no pobres la disponibilidad de dinero por un trabajo remunerado no se relacionó con ser fumador. Esto podría atribuirse a que un joven clasificado como no pobre tiene acceso a mayores recursos provenientes de su familia que podrían solventar su hábito. Por el contrario, en el grupo cercano a la línea de pobreza, en el cual los recursos familiares son más reducidos, se encontró una mayor posibilidad de que un joven fume si tiene dinero disponible proveniente de trabajo remunerado. En contraste, en el grupo de hogares pobres, el hecho de que el joven tenga un 


\section{Cuadro II}

Características socioeconómicas y demográficas RELACIONADAS CON EL CONSUMO DE TABACO

DE LOS JÓVENES PARTICIPANTES EN LA EVALUACIÓN BASAL del programa Oportunidades (México, 200I) $\begin{array}{ccc}\text { Fumador } & \text { Experimentador } & \text { No fumador } \\ (3.48 \%) & (9.89 \%) & \text { (86.63\%) }\end{array}$

Edad (\%)

\begin{tabular}{crrrr}
$10-14$ & 0.62 & 4.44 & 94.95 & $<0.01$ \\
\hline $15-18$ & 6.09 & 16.41 & 77.51 & \\
\hline $19-21$ & 9.44 & 18.14 & 72.42 & \\
\hline $\begin{array}{l}\text { Sexo (\%) } \\
\text { Mujeres }\end{array}$ & 1.19 & 7.95 & 90.87 & $<0.01$ \\
\hline Hombres & 6.22 & 12.22 & 81.56 & \\
$\begin{array}{l}\text { Clasificación } \\
\text { de pobreza (\%) } \\
\text { No pobre }\end{array}$ & 4.86 & 14.68 & 80.46 & $<0.01$ \\
\hline Cercano & 4.65 & 11.64 & 83.71 & \\
\hline Pobre & 2.91 & 8.60 & 88.49 &
\end{tabular}

Trabajo

remunerado $(\%)$

$<18$ años

\begin{tabular}{crrrr} 
Sí & 6.92 & 17.00 & 76.08 & $<0.01$ \\
\hline $\begin{array}{c}\text { No } \\
\geq 18 \text { años }\end{array}$ & 0.98 & 6.00 & 93.03 & \\
Sí & 15.07 & 23.12 & 61.81 & $<0.01$ \\
\hline No & 4.87 & 13.79 & 81.34 &
\end{tabular}

Tipo de escuela (\%)

\begin{tabular}{crrrr}
$\begin{array}{ccc}\text { Pública } \\
\text { Privada }\end{array}$ & 0.95 & 6.22 & 92.83 & $<0.01$ \\
\hline & 5.42 & 15.16 & 79.42 &
\end{tabular}

Asistencia

a la escuela (\%)

$<18$ años

\begin{tabular}{crrrr} 
Sí & 0.72 & 5.70 & 93.58 & $<0.01$ \\
\hline No & 6.57 & 15.97 & 77.45 & \\
$\geq 18$ años & & & & \\
Sí & 6.82 & 19.52 & 73.66 & 0.03 \\
\hline No & 9.93 & 17.97 & 72.10 &
\end{tabular}

*Nivel de significancia para una prueba de hipótesis (ji cuadrada) de no relación para datos agrupados por localidad de residencia.

trabajo remunerado fue un determinante marginal en su condición de fumador. Esta diferencia, en relación con los grupos menos desfavorecidos, podría atribuirse a la posibilidad de que en condiciones de extrema pobreza cualquier ingreso adicional disponible se destine a cubrir otros gastos familiares.

En el presente estudió se mostró una diferencia en el consumo de tabaco por género en los grupos de jóvenes con mayor edad (la posibilidad de ser fumador en relación con ser experimentador es mayor en el grupo de hombres que en el de mujeres); no obstante, los resultados de este estudio sugieren que esta

\section{Cuadro III}

Factores ReLACIONADOS CON EL CONSUMO dE TABACO (FUMADOR CONTRA EXPERIMENTADOR) EN 2912 JÓVENES PARTICIPANTES EN LA EVALUACIÓN BASAL DEL programa Oportunidades (México, 200I)

$\begin{array}{llll}\text { Variable } & R M M^{\S} & \mathrm{IC} 95 \% & \text { Valor } p\end{array}$

Edad (años)

\begin{tabular}{cccc}
$10-14^{\ddagger}$ & 1 & & \\
\hline $15-18$ & 1.6 & $0.9-3.0$ & 0.12 \\
\hline $19-21$ & 1.4 & $0.8-2.6$ & 0.28
\end{tabular}

Sexo

\begin{tabular}{crrr} 
Mujeres & $\ddagger$ & 1 & \\
\\
Hombres & 1.7 & $1.0-2.9$ & 0.04 \\
& & & \\
$\begin{array}{c}\text { Interacción sexo-edad } \\
10-14^{\ddagger}\end{array}$ & 1 & & \\
\hline $15-18$ & $1.5^{*}$ & $0.8-3.0$ & 0.21 \\
\hline $19-21$ & $2.5^{*}$ & $1.3-4.9$ & $<0.01$
\end{tabular}

Trabajo remunerado

$\begin{array}{lrrr}\mathrm{No}^{\ddagger} & \mathrm{I} & & \\ \mathrm{S} i ́ & 1.5 & 1.2-1.8 & <0.01\end{array}$

Nivel de pobreza

\begin{tabular}{lrrr} 
No pobre & & & \\
\hline Cercano & 1 & $0.9-1.6$ & 0.15 \\
\hline Pobre & 1.2 & $0.8-1.4$ & 0.75
\end{tabular}

Consumo de alcohol

$\begin{array}{lrrr}\begin{array}{c}\text { Consumo de alcohol } \\ \mathrm{No}^{\ddagger}\end{array} & \mathrm{I} & & \\ \mathrm{Sí} & 1.8 & 1.5-2.2 & <0.01 \\ \begin{array}{c}\text { Consumo de drogas } \\ \mathrm{No}^{\ddagger}\end{array} & \mathrm{I} & & \\ \mathrm{Sí} & 3.4 & 2.3-5.0 & <0.01\end{array}$

¥ Categoría de referencia.

*Razones de momios de hombres y mujeres en el grupo de edad correspondiente en relación con el grupo de edad de referencia. $\S R M$, razón de momios.

diferencia se reduce en los grupos más jóvenes. Más aún, la edad de inicio del consumo habitual de tabaco en esta población fue muy similar en hombres y mujeres (14.8 y 14.6 años, respectivamente). Estos resultados son consistentes con los estudios que han documentado que el hábito tabáquico es mayor en hombres, ${ }^{1}$ pero también con aquellos que sugieren que en los países en desarrollo esta diferencia tiende a reducirse en los grupos más jóvenes. ${ }^{16}$ Las edades de inicio reportadas confirman los resultados que documentaron Campuzano y colaboradores, quienes mostraron que en la población urbana mexicana la edad media para iniciar el consumo de tabaco bajó de 21.8 años a 13.2 años en cinco décadas. ${ }^{10}$

Es sabido que la mayoría de los fumadores empieza su adicción antes de los 20 años y el inicio temprano 


\section{Cuadro IV \\ FACTORES RELACIONADOS CON EL CONSUMO de TABACO (FUMADOR CONTRA EXPERIMENTADOR) SEGÚN LA

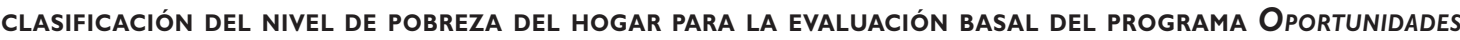 \\ (MÉXICO, 200I)}

\begin{tabular}{|c|c|c|c|}
\hline & Modelo I: Hogares no pobres & $\begin{array}{l}\text { Modelo 2: Hogares cercanos } \\
\text { a la línea de pobreza }\end{array}$ & Modelo 3: Hogares pobres \\
\hline Variable & $\begin{array}{l}R M^{*} \\
(I C 95 \%)\end{array}$ & $\begin{array}{cc}R M^{*} & \text { Valor } p \\
(I C 95 \%) & \end{array}$ & $\begin{array}{c}R M^{*} \\
(I C 95 \%)\end{array}$ \\
\hline
\end{tabular}

\begin{tabular}{|c|c|c|c|c|c|c|}
\hline \\
\hline $10-14^{\ddagger}$ & \multicolumn{2}{|l|}{ I } & \multicolumn{2}{|l|}{ I } & \multicolumn{2}{|l|}{ I } \\
\hline $15-18$ & $3.5(0.4-28)$ & 0.2 & $1.6(0.4-5.4)$ & 0.4 & $1.4(0.7-2.8)$ & 0.3 \\
\hline$|9-2|$ & $1.3(0.1-13)$ & 0.8 & $1.5(0.4-5.4)$ & 0.6 & $1.5(0.7-3.1)$ & 0.3 \\
\hline
\end{tabular}

\begin{tabular}{|c|c|c|c|c|c|c|}
\hline Sexo & & & & & & \\
\hline Mujeres ${ }^{\ddagger}$ & I & & I & & 1 & \\
\hline Hombres & $5.1(2.8-9.2)$ & $<0.01$ & $2.6(1.5-4.3)$ & $<0.01$ & $3.0(2.2-4.2)$ & $<0.01$ \\
\hline \multicolumn{7}{|c|}{ Trabajo remunerado } \\
\hline $\mathrm{No}^{\ddagger}$ & I & & I & & I & \\
\hline Sí & $1.3(0.7-2.2)$ & 0.39 & $2.2(1.5-3.2)$ & $<0.01$ & $1.2(0.9-1.6)$ & 0.1 \\
\hline
\end{tabular}

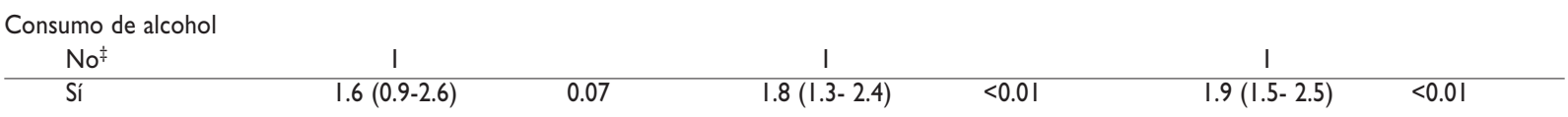

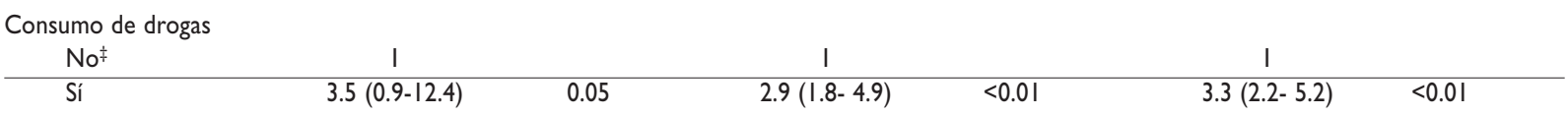

\begin{tabular}{llccc}
$\begin{array}{l}\text { Autoestima } \\
\text { Normal }\end{array}$ & - & I & & \\
\hline Baja & - & $2.5(1.2-5.1)$ & 0.02 & -
\end{tabular}

$\begin{aligned} & \text { Apoyo emocional } \\ & \text { No }\end{aligned}$
Sí

\begin{tabular}{llllr}
$\begin{array}{l}\text { Interacción sexo-edad } \\
10-14^{\ddagger}\end{array}$ & - & \multicolumn{2}{l}{} \\
\hline $15-18$ & - & - & $1.8^{*}(0.8-3.8)$ & 0.2 \\
\hline $19-21$ & - & - & $2.5^{*}(1.1-5.7)$ & 0.04
\end{tabular}

¥ Categoría de referencia.

* RM de grupo de edad correspondiente en comparación con la edad de referencia en ambos sexos.

predice: a) una duración del consumo más larga, b) una frecuencia de consumo diario mayor y c) una mayor probabilidad de dependencia. ${ }^{20-23}$ En consecuencia, un programa nacional para controlar el tabaquismo debe tener como objetivo principal prevenir el inicio del tabaquismo, en particular entre los jóvenes.

En países o ciudades con niveles de pobreza elevados, el consumo de tabaco tiene dos efectos. El primero es el ya conocido de propiciar adicción y daños directos a la salud; el otro es el desplazamiento de otros bienes necesarios para el desarrollo de la familia, como son los alimentos y la inversión en educación. Se ha documentado una concentración del consumo de tabaco en población de nivel socioeconómico bajo y un incremento del riesgo para iniciar su consumo, ${ }^{21}$ en países en desarrollo en los que las familias destinan entre 5.5 y $17 \%$ de su gasto al tabaco, con desplazamiento incluso de la compra de otros bienes fundamentales, como los alimentos. ${ }^{22,23}$ En realidad, en hogares de nivel socioeconómico bajo, expresado como escaso nivel educativo, el gasto en tabaco es $34 \%$ superior que en hogares de mayor nivel socioeconómico. ${ }^{24}$ Un hallaz- 
go importante de este estudio es que la prevalencia del consumo de tabaco es menor en los jóvenes que residen en hogares calificados como pobres; en cambio, si tienen un ingreso adicional por trabajo, la probabilidad de consumo se incrementa.

Dado que el programa Oportunidades incluye transferencias monetarias directas a los hogares incorporados al Programa, los resultados alertan sobre la necesidad de incorporar campañas de prevención para evitar que la disponibilidad adicional de dinero en los jóvenes se utilice en la compra de cigarrillos.

El estudio tiene las limitaciones propias de los diseños transversales, y los efectos notificados deben interpretarse como relaciones entre las variables de estudio y no como las relaciones causales. Asimismo, la posible presencia de algún adulto al momento de aplicar la encuesta la encuesta podría inhibir 1 a respuesta del joven y como consecuencia tener una subestimación de la frecuencia de consumo que informó el joven, ya que es un comportamiento censurado y por lo tanto no puede registrarse con veracidad.

Se ha documentado que el consumo de tabaco inicia en la adolescencia temprana y casi toda la experimentación tiene lugar antes de concluir la preparatoria. ${ }^{8}$ Algunos estudios efectuados en Estados Unidos de América han documentado que el riesgo de fumar en el adolescente es mayor cuando coinciden factores como padres que fuman, hogares de bajos ingresos o bajo nivel educativo. Por otro lado, una investigación en estudiantes universitarios en México detectó incidencias más altas de inicio de consumo de tabaco para ambos sexos entre los estudiantes de mayor nivel socioeconómico. ${ }^{25}$ Las encuestas nacionales de adicciones de 1988 y 1993 indicaron que la curiosidad (46.9 y $45 \%$, respectivamente) y la presión de los amigos (34.6 y $37 \%$, respectivamente) fueron los motivos más frecuentes para iniciar el consumo de tabaco.

En adolescentes se han identificado otros factores vinculados con el tabaquismo, entre ellos los siguientes: a) individuales, como género masculino, ser menor de edad, bajo nivel educativo y cambios en las condiciones de vida; b) socioeconómicos, como desigualdad económica y disponibilidad de dinero; c) familiares, como la presencia de un familiar fumador, $y$ alteraciones emocionales adversas, como rupturas familiares y falta de apoyo de los padres; y d) condiciones sociales, como regulación, promoción e influencia de los medios masivos de comunicación. ${ }^{14}$

De acuerdo con lo reportado en otros estudios, la mayoría de los jóvenes fumadores desea dejar de fumar y más de dos terceras partes lo han intentado, ${ }^{13}$ pero la nicotina presente en los cigarrillos crea adicción física y psicológica, por lo que no resulta fácil in- terrumpir su consumo. Por lo tanto, la generación de conocimiento sobre los determinantes de consumo de tabaco en jóvenes posibilitará planear políticas dirigidas a la prevención del hábito de fumar y diseñar programas adecuados para abandonar el hábito .

Este estudio ha permitido caracterizar algunos factores que se vinculan con el patrón de consumo de tabaco, en especial la transición de experimentar a fumar. El acceso de los jóvenes al tabaco se facilita gracias a la actividad mercadotécnica que lleva a cabo la industria (se invierten un poco más de 5000 millones de dólares anuales para promocionar, mantener e incrementar el uso del tabaco ${ }^{2}$ ), la ausencia de programas educativos dirigidos a jóvenes y la falta del cumplimiento de la normatividad relacionada con el control del tabaquismo. ${ }^{7}$

\section{Referencias}

I. Mackay J, Eriksen M. The tobacco atlas. Geneva:World Health Organization, 2002.

2. U.S. Department of Health and Human Services. Reducing tobacco use: a report of the surgeon general. Atlanta, Georgia: U.S. Department of Health and Human Services, Centers for Disease Control and Prevention, National Center for Chronic Disease Prevention and Health Promotion, Office on Smoking and Health, 2000.

3. Tapia-Conyer R, Kuri-Morales P, Hoy-Gutiérrez MJ. Panorama epidemiológico del tabaquismo en México. Salud Publica Mex 200I;43:478-484.

4. Gilmore A, Pomerleau J, McKee $M$ et al. Prevalence of smoking in 8 countries of the former Soviet Union: results from the living conditions, lifestyles and health study. Am J Public Health 2004;94(I2):2177-87.

5. Rojas G, Gaete J, González I et al.Tabaquismo y salud mental. Rev Med Chil 2003;13I(8):873-880.

6. Meneses-González F, Márquez-Serrano M, Sepúlveda-Amor J et al. La industria del tabaco en México. Salud Publica Mex 2002;44(supl I): SI6ISI69.

7. DiFranza JR, Tye JB. Who profits from tobacco sales to children? JAMA 1990;263(20):2784-7.

8. Tyas SL, Pederson LL. Psychosocial factors related to adolescent smoking: a critical review of the literature. Tob Control 1998;7(4):409-420.

9.American Cancer Society. Tobacco control country profiles. In: Shafey O, Dolwick S, Guindon E, eds. 2da. edición Atlanta, GA:American Cancer Society 2003.

10. Campuzano-Rincón JC, Hernández-Avila M, Sammet JM, MéndezRamírez I, Tapia-Conyer R, Sepúlveda-Amor J. Comportamiento de los fumadores en México según las Encuestas Nacionales de Adicciones 1988 a 1998. Primer Informe sobre el combate al tabaquismo en México. En: Valdés-Salgado R, Lazcano-Ponce E, Hernández-Avila M, ed. México, Cuernavaca: Instituto Nacional de Salud Pública, 2005:21-27.

I I. Comité Técnico para la Medición de la Pobreza. Medición de la Pobreza.Variantes metodológicas y estimación preliminar. México: SEDESOL, 2002.

12. Glass GV, Stanley JC. Métodos estadísticos aplicados a las ciencias sociales. Madrid: Prentice Hall Internacional, 1974:7.

13. Siegel S. Estadística no paramétrica. México:Trillas, |991:74-79. 
I4.Arillo-Santillán E, Fernández E. Prevalencia de tabaquismo y bajo desempeño escolar, en estudiantes de II a 24 años de edad del estado de Morelos, México. Salud Publica Mex 2002;144:54-66.

15. Primer Informe sobre el Combate al Tabaquismo en México: México, Cuernavaca: Instituto Nacional de Salud Pública, 2005:43-46.

16. Torrecilla GM, Ruano GR, Plaza MD et al. Jóvenes y tabaco: prevalencia, patrón y actitud ante el consumo de tabaco. An Pediatr (Barc) 2004;60(5):440-449.

17. Banks MH, Bewlel BR. Long-term study of smoking by secondary schoolchildren. Arch Dis Child 1978;53:12-19.

18. Oakley A, Brannen J.Young people, gender and smoking in the United Kingdom. Health Promot Int 1992;7:75-88.

19. Hammarstrom A, Janlert U. Unemployment and change of tobacco habits: a study of young people from 16-21 years of age. Addiction 1994;89:1691-1696.

20. Breslau N, Peterson EL. Smoking cessation in young adults: age at initiation of cigarette smoking and other suspected influences. Am J Public Health 1996;86(2):214-220.
21. Gilman SE, Abrams DB, Buka SL. Socioeconomic status over the life course and stages of cigarette use: initiation, regular use, and cessation.J Epidemiol Community Health 2003;57:802-808. 22. De Beber J, Lovelace C, Yurekli A. Poverty and tobacco.Tob Control 2001;10:210-2II.

23. Efroymson D, Ahmed S, Townsend J et al. Hungry for tobacco: an analysis of the economic impact of tobacco consumption on the poor in Bangladesh. Tob Control 2001;10:212-217.

24. Siahpush M. Socioeconomic status and tobacco expenditure among Australian households: results from the 1998-99 Household Expenditure Survey.J Epidemiol Community Health 2003;57:798-80I.

25. Arillo-Santillán E, Lazcano-Pnce E, Sánchez-Zamorano LM, ValdésSalgado R, Fernández E, Sammet JM et al. Estudio poblacional de tabaquismo en adolescentes de Morelos. Factores asociados a la prevalencia, incidencia, persistencia y cesación, Primer Informe sobre el combate al tabaquismo en México. En:Valdés-Salgado R, Lazcano-Ponce E, Hernández-Avila M, ed. 1998-2003. México. Cuernavaca: Instituto Nacional de Salud Pública, 2005:47-64. 